\title{
Correction to: Principles of Neuro-Oncology
}

Alejandro Monroy-Sosa, Srikant S. Chakravarthi, Jaime G. de la Garza-Salazar, Abelardo Meneses Garcia, and Amin B. Kassam

\section{Correction to: Mapping the Brain During Awake Surgery for Patients with Neurological Disorders Including Brain Tumors: Henry Ruiz-Garcia, Erik Middlebrooks, David Sabsevitz, Perry Bechtle, and Alfredo Quinones-Hinojosa, https://doi.org/10.1007/978-3-030-54879-7_21}

The chapter was published inadvertently with the wrong video legends and now the legends are updated as below:

Video 21.1 Surgical technique for awake mapping in a case of high-grade glioma patient

Video 21.2 Three-dimensional reconstruction of the tumor and simulated white matter tracts around the lesion

And Video 21.2 was uploaded incorrectly in the previous version and the correct video was embedded in this version of the book.

\section{Correction to: Endoscopic Endonasal Far Medial Approach: Luis C. Requena Requena, Samuel Viera, Alexandre B. Todeschini, Thiago A. Feliccio, Douglas A. Hardesty, Brad A. Otto, Ricardo L. Carrau, Daniel M. Prevedello, https://doi.org/10.1007/978-3-030-54879-7_40}

The book was inadvertently published with an incorrect spelling of the author's name in the Chapter as Douglas A. Hardsesty whereas it should be Douglas A. Hardesty. This error has now been corrected with this erratum.

\footnotetext{
The updated version of the chapter can be found at https://doi.org/10.1007/978-3-030-54879-7_21 https://doi.org/10.1007/978-3-030-54879-7_40
} 